\title{
Implementation of Bubble CPAP in a Rural Ugandan Neonatal ICU
}

\author{
Ryan M McAdams MD, Anna B Hedstrom MD, Robert M DiBlasi RRT-NPS FAARC, \\ Jill E Mant MBBS MA MRCPCH, James Nyonyintono MB ChB, Christine D Otai RNM, \\ Debbie A Lester RN IBCLC, and Maneesh Batra MD MPH
}

\begin{abstract}
BACKGROUND: Respiratory distress is a leading cause of neonatal death in low-income and middle-income countries. CPAP is a simple and effective respiratory support modality used to support neonates with respiratory failure and can be used in low-income and middle-income countries. The goal of this study was to describe implementation of the Silverman-Andersen respiratory severity score (RSS) and bubble CPAP in a rural Ugandan neonatal NICU. We sought to determine whether physicians and nurses in a low-income/middle-income setting would assign similar RSS in neonates after an initial training period and over time. METHODS: We describe the process of training NICU staff to use the RSS to assist in decision making regarding initiation, titration, and termination of bubble CPAP for neonates with respiratory distress. Characteristics of all neonates with respiratory failure treated with bubble CPAP in a rural Ugandan NICU from January to June 2012 are provided. RESULTS: Nineteen NICU staff members (4 doctors and 15 nurses) received RSS training. After this, the Spearman correlation coefficient for respiratory severity scoring between doctor and nurse was 0.73 . Twenty-one infants, all $<3 \mathrm{~d}$ of age, were treated with CPAP, with 17 infants starting on the day of birth. The majority of infants $(16 / 21$, $76 \%$ ) were preterm, $10(48 \%)$ were $<1,500 \mathrm{~g}$ (birthweight), and $13(62 \%)$ were outborn. The most common diagnoses were respiratory distress syndrome $(16 / 21,76 \%)$ and birth asphyxia $(5 / 21$, $24 \%$ ). The average RSS was $7.4 \pm 1.3$ before starting CPAP, $5.2 \pm 2.3$ after $2-4 \mathrm{~h}$ of CPAP, $4.9 \pm 2.7$ after $12-24 \mathrm{~h}$ of CPAP, and $3.5 \pm 1.9$ before CPAP was discontinued. Duration of treatment with CPAP averaged $79 \pm 43 \mathrm{~h}$. Approximately half $(11 / 21,52 \%)$ of infants treated with CPAP survived to discharge. CONCLUSIONS: Implementing bubble CPAP in a low-income/middle-income setting is feasible. The RSS may be a simple and useful tool for monitoring a neonate's respiratory status and for guiding CPAP management. Key words: low-income and middle-income countries; neonate; respiratory distress; respiratory severity score. [Respir Care 2015;60(3):437-445. (C) 2015 Daedalus Enterprises]
\end{abstract}

\section{Introduction}

Preterm birth remains a major cause of neonatal mortality globally. ${ }^{1}$ Respiratory distress syndrome (RDS) is a

Drs McAdams, Hedstrom, and Batra are affiliated with the Department of Pediatrics, University of Washington, Seattle, Washington. Drs McAdams, Hedstrom, and Batra and Mr DiBlasi are affiliated with the Department of Pediatrics, Seattle Children's Hospital, Seattle, Washington. Ms Mant is affiliated with the Paediatrics Department, York Hospital, York, United Kingdom. Mr Nyonyintono and Ms Otai are affiliated with Pediatrics, Kiwoko Hospital, Luweero, Uganda. Ms Lester is affiliated with The ISIS Foundation, Edmonds, Washington.

The authors have disclosed no conflicts of interest. Mr DiBlasi has relationships with Dräger, Ikaria, and Vapotherm. common and serious complication of premature birth, resulting from surfactant deficiency and structural immaturity of the lungs. In high-income countries, effective strategies to treat RDS, such as exogenous surfactant replacement therapy, CPAP, and mechanical ventilation have resulted in markedly improved survival for preterm in-

\footnotetext{
Dr Batra presented a version of this paper at the AARC Congress 2012, held November 10-13, 2012, in New Orleans, Louisiana.

Correspondence: Ryan M McAdams MD, Department of Pediatrics, Division of Neonatology, University of Washington, Box 356320, Seattle, WA 98195-6320. E-mail: mcadams@uw.edu.
}

DOI: $10.4187 /$ respcare. 03438 
fants. $^{2-4}$ Until recently, these therapies have not been routinely available in low-income and middle-income countries due to the cost and complexity of implementation.

CPAP is a well-established and effective therapy for RDS. Several studies have shown that early CPAP can effectively treat RDS without invasive mechanical ventilation or surfactant therapy in preterm infants..$^{5-7}$ Ventilators and exogenous surfactant therapy are expensive, have the potential for life-threatening complications, and require placement of an endotracheal tube that requires significant technical expertise. ${ }^{8}$ Although expensive CPAP devices exist in high-resource settings, provision of CPAP can be achieved with little technology or cost. The components of CPAP include a gas source, nasal or facemask airway interface, and a pressure-generating apparatus. Oxygen concentrators can provide sufficient operational flow and, along with low-resistance nasal cannula interfaces, are readily available in many low-income and middle-income countries. ${ }^{9}$ Tubing submerged in a bottle of water can serve as the pressure valve, with the depth of the tubing in the water determining the pressure in the circuit. An example of this simple noninvasive ventilation method is bubble CPAP, which has been safely and effectively used in low-income and middle-income countries to improve survival in infants with many forms of neonatal respiratory distress. ${ }^{10-12}$

CPAP applied to immature lungs helps to increase functional residual capacity, improve chest wall stability, and decrease work of breathing, thereby reducing the need for mechanical ventilation and surfactant administration. ${ }^{13}$ In high-income settings, deciding which patients will benefit from CPAP is often based on clinical exam, breathing frequency, $\mathrm{S}_{\mathrm{pO}_{2}}$, blood gas measurements, and chest radiograph. In low-income/middle-income settings, lack of blood gas machines and x-ray capability can hinder decision making regarding proper treatment modalities for neonatal respiratory distress. In these resource-limited settings, a clinical assessment tool that provides accurate and reliable assessment of a neonate's respiratory status may appropriately guide clinical management. The SilvermanAndersen respiratory severity score (RSS) ${ }^{14}$ is a validated bedside exam method for assessing the degree of respiratory distress in neonates and can be used in any resource setting.

We describe training using the Silverman-Andersen RSS bedside tool and implementation of an inexpensive bubble CPAP system in a rural neonatal ICU (NICU) in Uganda. We sought to determine whether physicians and nurses in a low-income/middle-income setting would assign similar RSS in neonates after an initial training period and over time. We report the physiologic responses to therapy and outcomes for the first 6 months after bubble CPAP implementation.

\section{QUICK LOOK}

\section{Current knowledge}

Respiratory distress syndrome (RDS) is a common and serious complication of premature birth, resulting from surfactant deficiency and structural immaturity of the lungs. In high-income countries, effective strategies to treat RDS, such as exogenous surfactant replacement therapy, CPAP, and mechanical ventilation, have resulted in markedly improved survival.

\section{What this paper contributes to our knowledge}

Implementing bubble CPAP in low-income/middle-income settings is feasible. The Silverman-Andersen respiratory severity score may be a simple and useful tool for monitoring respiratory status and for guiding CPAP management.

\section{Methods}

\section{Setting}

The NICU at Kiwoko Hospital is a referral center for 3 rural districts of central Uganda with a combined population of 600,000 . As of the 2002 census, $89.5 \%$ of the population was rural dwelling. ${ }^{15}$ In 2006 , the estimated neonatal and child mortality rates in this region were 33 and 129 per 1,000 live births, respectively. ${ }^{16}$ The majority of mothers $(69.1 \%)$ deliver in a health-care facility, ${ }^{16}$ but referral systems for pregnant mothers at risk for birth complications are lacking. The majority of pregnant mothers are transferred to the hospital by motorcycle or taxi.

Kiwoko Hospital, which has 2,100 births annually, provides the only neonatal intensive care services in the region and admitted 650 infants in 2012. The NICU staff consists of an assigned physician who sees all patients daily and a pool of 23 nurses; $2-4$ nurses are assigned per shift. During the period of this study, the average NICU census was 22 , with a patient-nurse ratio of 5:1. There are no laboratory blood gas analyzers available at the hospital, and $\mathrm{x}$-ray capability is limited. Surfactant therapy was not available at Kiwoko Hospital during the study period. Before January 2012, the only treatment available for neonates with respiratory distress was supplemental oxygen provided via nasal cannula and oxygen concentrator.

\section{RSS Training}

Before the introduction of bubble CPAP, no standardized method to assess neonatal respiratory status existed in the Kiwoko Hospital NICU. The RSS evaluates 5 param- 


\section{RESPIRATORY SEVERITY SCORE}

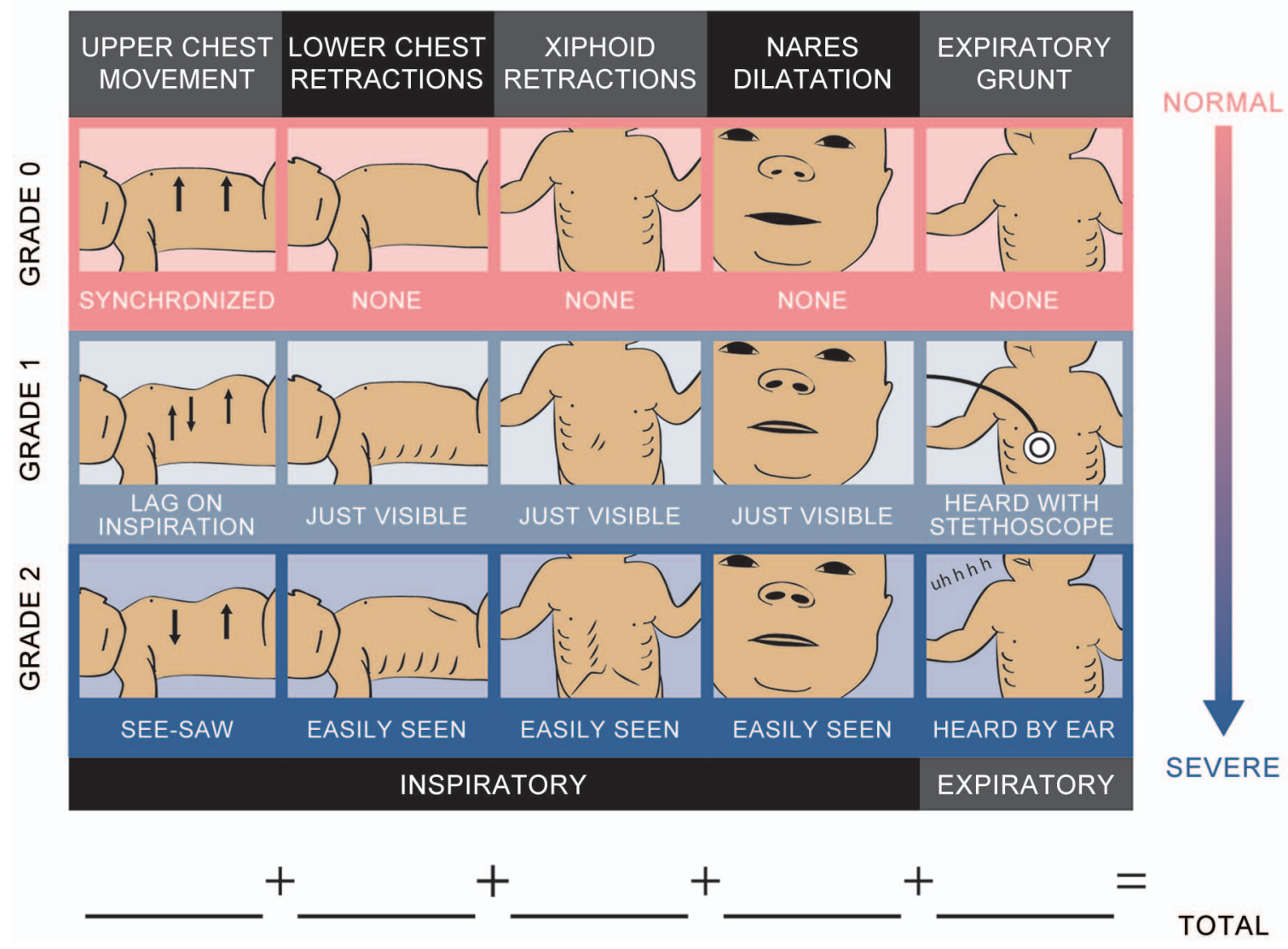

Fig. 1. The respiratory severity score for assessing the degree of respiratory distress in neonates. Modified from reference 14 .

eters and assigns a numerical score for each parameter, with 0 being the best score possible in each category and 2 being the worst (Fig. 1). Parameters assessed are: upper chest wall movement compared with abdominal movement, lower chest retractions, xiphoid retractions, nasal flaring (nares dilatation), and expiratory grunting. Healthy neonates without increased work of breathing have a cumulative score close to 0 , whereas neonates with severely increased work of breathing have scores closer to 10 . NICU nurses and physicians were trained on the RSS over the course of 1 week in January 2012 through a series of educational workshops while on the job.

Inter-rater reliability testing regarding RSS was conducted 3 months after the initial RSS training. NICU nurses and the sole physician on duty in the NICU simultaneously recorded breathing frequency and RSS scores on all subjects $(n=38)$ in the NICU in a blinded fashion. Breathing frequency and RSS were analyzed using Pearson and Spearman correlation coefficients, respectively. Nurses and doctors $(n=11)$ also scored a video demonstrating a subject with more severe distress (RSS of 8). Scores of this video were categorized as high $(>7)$, medium (5-7), and low
$(<5)$ to evaluate the proportion that could correctly identify severe distress.

\section{CPAP Training and Implementation}

The 2 local physicians responsible for the NICU, a pediatrician (JEM) and a surgeon (JN) with experience caring for neonates, were trained on how to set up and use the bubble CPAP device. Nurses and physicians were trained on the technical aspects of the device, including instruction on bubble CPAP system maintenance and how to monitor for proper device performance. During this training, bubble CPAP was used for the first time on 2 babies in the NICU. After the initial training, NICU nurses and staff received ongoing feedback and hands-on training for an additional 1 week.

The NICU nursing staff performed serial RSS measurements during routine collection of vital signs on all neonates admitted with respiratory distress. Selection for bubble CPAP treatment was at the discretion of 2 local physicians based on the degree of a neonate's respiratory failure and perceived probability of death without CPAP. 


\section{BubBle CPAP in a RuRal Ugandan NICU}
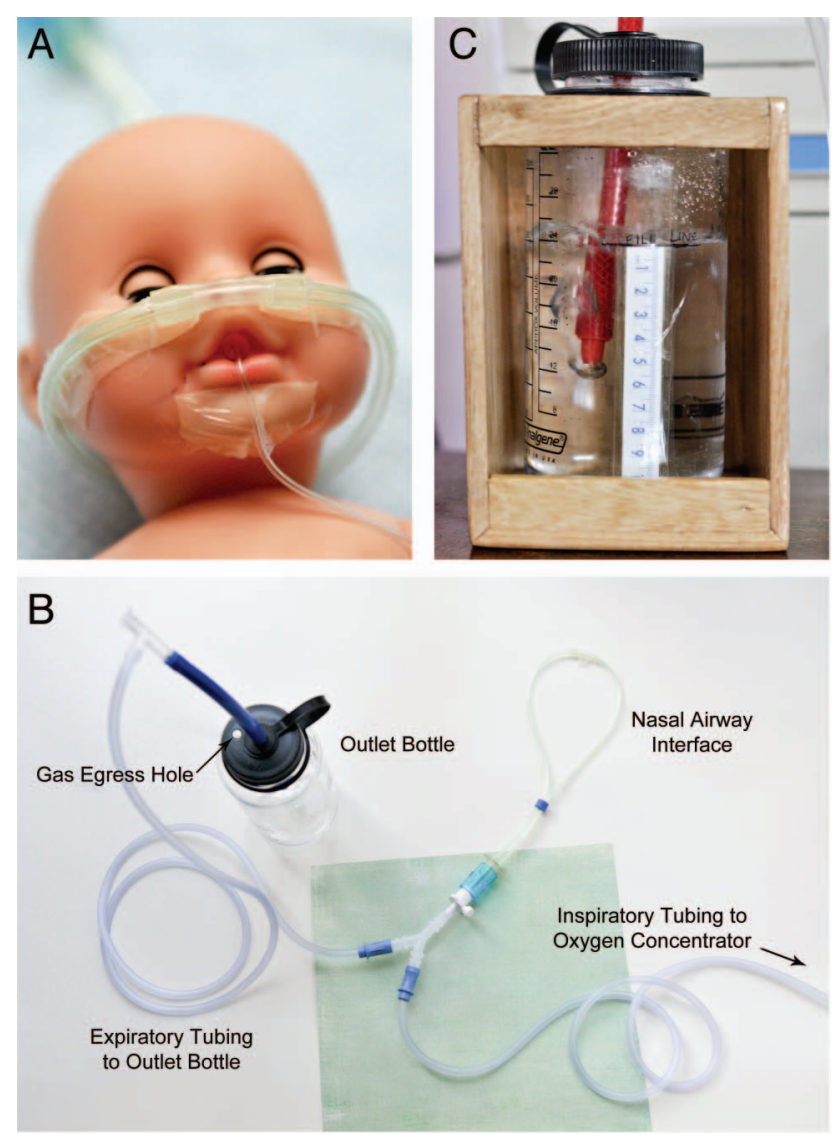

Fig. 2. Bubble CPAP set-up. A: The airway interface consisted of an RAM Cannula with occlusive prongs secured to the face with tape. An orogastric tube should be placed for gastric decompression. B: The bubble CPAP system consisted of a simple water-seal column (outlet bottle), bubbler tube, inspiratory and expiratory tubing, and connectors, which were adapted in series. C: An improvised wooden case allowed the outlet bottle to remain in an upright position.

Admission diagnosis, $\mathrm{S}_{\mathrm{pO}_{2}}$, breathing frequency, RSS, treatment duration, and reason for discontinuing CPAP, which included mortality, were analyzed for all neonates treated with bubble CPAP during the study period.

\section{Equipment}

The Kiwoko Hospital NICU had no pressurized air or oxygen gas source; the only available oxygen was provided by concentrators (DeVilbiss Healthcare, Somerset, Pennsylvania). Oxygen blenders and mechanical ventilators were not available, but pulse oximeters were used for 3 y before CPAP implementation.

The airway interface consisted of a nasal cannula (Neotech RAM Cannula, Neotech, Valencia, California) with occlusive prongs (Fig. 2A) that could be fastened to the face similar to a nasal cannula. The tubing diameter of the RAM Cannula is larger than that provided by a standard oxygen cannula and is similar to nasal CPAP prongs, which allow low resistance to breathing and adequate pressure delivery to the nasal airway opening. The bubble CPAP system consisted of a simple water-seal column (outlet bottle), bubbler tube, inspiratory and expiratory tubing, and connectors, which were adapted in series (Fig. 2B). The depth of the distal bubbler below the water surface, as determined via a measuring tape on the outside of the bottle, corresponded with the desired CPAP level $\left(5 \mathrm{~cm}=5 \mathrm{~cm} \mathrm{H}_{2} \mathrm{O}\right)$. Before implementation, the system was attached to a spontaneously breathing neonatal test lung/nasal airway model, and the preset pressures coincided with the delivered lung pressures (within $1 \mathrm{~cm} \mathrm{H}_{2} \mathrm{O}$; data not shown) at multiple CPAP levels and flows. An improvised wooden case (Fig. 2C) allowed the outlet bottle to remain in an upright position.

Before each use of the CPAP device, the system was inspected for any visible soiling or defects and was washed with a diluted bleach cleaning solution (eg, regular JIK, Reckitt Benckiser, Nairobi, Kenya). Flow from the oxygen concentrator was adjusted so that bubbling in the outlet bottle was maintained throughout the entire respiratory cycle. Upon initiation of bubble CPAP, an orogastric tube, vented to atmosphere, was placed to prevent gastric insuf- 


\section{Bubble CPAP in a RuRAl Ugandan NICU}

flation. $\mathrm{S}_{\mathrm{pO}_{2}}$ and heart rates were recorded using a Nellcor 560 pulse oximeter (Covidien, Boulder, Colorado).

\section{Case Series and Implementation}

All neonates admitted to the NICU and treated with bubble CPAP from January to June 2012 were included in this study. Neonates were eligible for bubble CPAP at the discretion of the attending physician, with no set exclusion criteria. During the RSS training period, attending physicians considered bubble CPAP only for infants with an RSS if $>5$ and if they thought that the infant would benefit from CPAP. Before initiating bubble CPAP, the NICU physician discussed the baby's RSS and reasons for or against CPAP with the nursing staff. Titration of CPAP was recommended based on work of breathing, which was assessed using the RSS. Data were collected prospectively and then de-identified before analysis. Extracted data included: birth or admission weight, diagnoses, gestational age, and corrected gestational age at the time CPAP was initiated. Respiratory-related physiologic data were obtained at 4 time points of interest: (1) the time that CPAP was started (baseline), (2) between 2 and $4 \mathrm{~h}$ after initiation, (3) 12-24 h after initiation, and (4) on the final day CPAP was administered (end point). At each of these time points, the following information was recorded: breathing frequency, $\mathrm{S}_{\mathrm{pO}_{2}}$, RSS, level of CPAP $\left(\mathrm{cm} \mathrm{H}_{2} \mathrm{O}\right)$, gas flow (L/min), complications (eg, nasal irritation), number of subjects in the unit, and number of nurses on duty. Nasal irritation (nasal erythema, mucosal erosion, or edema) was determined by visual inspection performed during each nursing shift while the subject was treated with bubble CPAP. Weaning to nasal cannula or room air was encouraged once infants were stable on a CPAP of $<5$ with an RSS of $<5$. Infants were weaned off CPAP at the discretion of the attending physician. The reason for stopping CPAP and the outcome of the neonate were recorded. The Seattle Children's Hospital institutional review board approved this study, with local approval obtained from Kiwoko Hospital.

\section{Results}

\section{RSS Reliability}

Nineteen NICU staff (4 doctors and 15 nurses) were trained on RSS. During the testing period, the NICU physician scored 38 NICU subjects simultaneously with nurses. Breathing frequency ranged from 24 to 80 breaths/min. The Pearson correlation coefficient for breathing frequency between doctor and nurse was 0.86 (Fig. 3A). The RSS of these same subjects ranged from 0 to 7 . Nurses had the same score as the physician $45 \%$ of the time, were within 1 point $87 \%$ of the time, and were within 2 points $100 \%$ of the time. The Spearman correlation coefficient for respiratory severity scoring between doctor and nurse was 0.73 (Fig. 3B).

A video showing a baby with respiratory distress (RSS of 8 ) was viewed and scored by 4 physicians and 8 nurses (median score of 8 , range of 7-10). The majority $(91.7 \%$, $11 / 12)$ of scorers gave this video a high score $(>7)$, whereas $8.3 \%(1 / 12)$ gave a medium score (between 5 and 7 ).

\section{Case Series}

During the study period, the average NICU census was $22 \pm 3$ (mean $\pm \mathrm{SD}$ ) subjects, with $4 \pm 1$ nurses (5:1 patient-to-nurse ratio) and one on-call physician per shift. Twenty-one infants were treated with CPAP during the study period, all starting at $<3 \mathrm{~d}$ of age, with 17 (80.9\%) infants starting on the day of birth (Table 1). Most infants, 91\% (19/21) were singletons, 76\% (16/21) were preterm, $48 \%(10 / 21)$ were very low birthweight $(<1,500 \mathrm{~g})$, and $62 \%$ (13/21) were born outside Kiwoko Hospital. Mean birthweight was $1,790 \mathrm{~g}$ (range of 1,070-3,460 g), and the mean estimated gestational age was 33.3 weeks (range of 28-41 weeks). The most common clinically determined diagnoses were RDS $(76 \%, 16 / 21)$ and birth asphyxia $(24 \%, 5 / 21)$.

Before starting CPAP, 20 of 21 infants were on nasal cannula delivering $100 \%$ oxygen, most commonly at a flow of 2-5 L. CPAP treatment was associated with a decrease in breathing frequency and an increase in $\mathrm{S}_{\mathrm{pO}_{2}}$ (Table 2). The average $\mathrm{S}_{\mathrm{pO}_{2}}$ at baseline was $86 \%$ (mean), with $62 \%$ (13/21) of infants noted to be tachypneic (breathing frequency of $>60$ breaths $/ \mathrm{min}$ ). The average RSS was $7.4 \pm 1.3$ before starting CPAP, $5.2 \pm 2.3$ after $2-4 \mathrm{~h}$ of CPAP, $4.9 \pm 2.7$ after $12-24 \mathrm{~h}$ of CPAP, and $3.5 \pm 1.9$ before CPAP was discontinued. Duration of treatment with CPAP averaged $79.1 \pm 42.8 \mathrm{~h}$. After treatment, 10 of 21 infants weaned to low-flow $(\leq 1 \mathrm{~L} / \mathrm{min}$ ) nasal cannula, and 2 came off respiratory support completely. Nine of 21 infants died on CPAP, and therefore, approximately half $(52.4 \%, 11 / 21)$ of infants treated with CPAP survived to discharge. Neonates treated with bubble CPAP who were diagnosed with RDS had a $62.5 \%$ (10/16) survival rate compared with a $40 \%(2 / 5)$ survival rate in neonates diagnosed with birth asphyxia. Although respiratory failure may have contributed to the lack of survival in the 9 neonates who died, the definitive cause of death is unknown since none of the neonates had autopsies performed. Blood cultures were not available at Kiwoko Hospital, so sepsis could not be ruled out as a causative factor.

No major complications of CPAP occurred during the study period. Only 3 (14\%) neonates were diagnosed with nasal irritation after bubble CPAP was started. 


\section{BubBle CPAP in a RuRal Ugandan NICU}
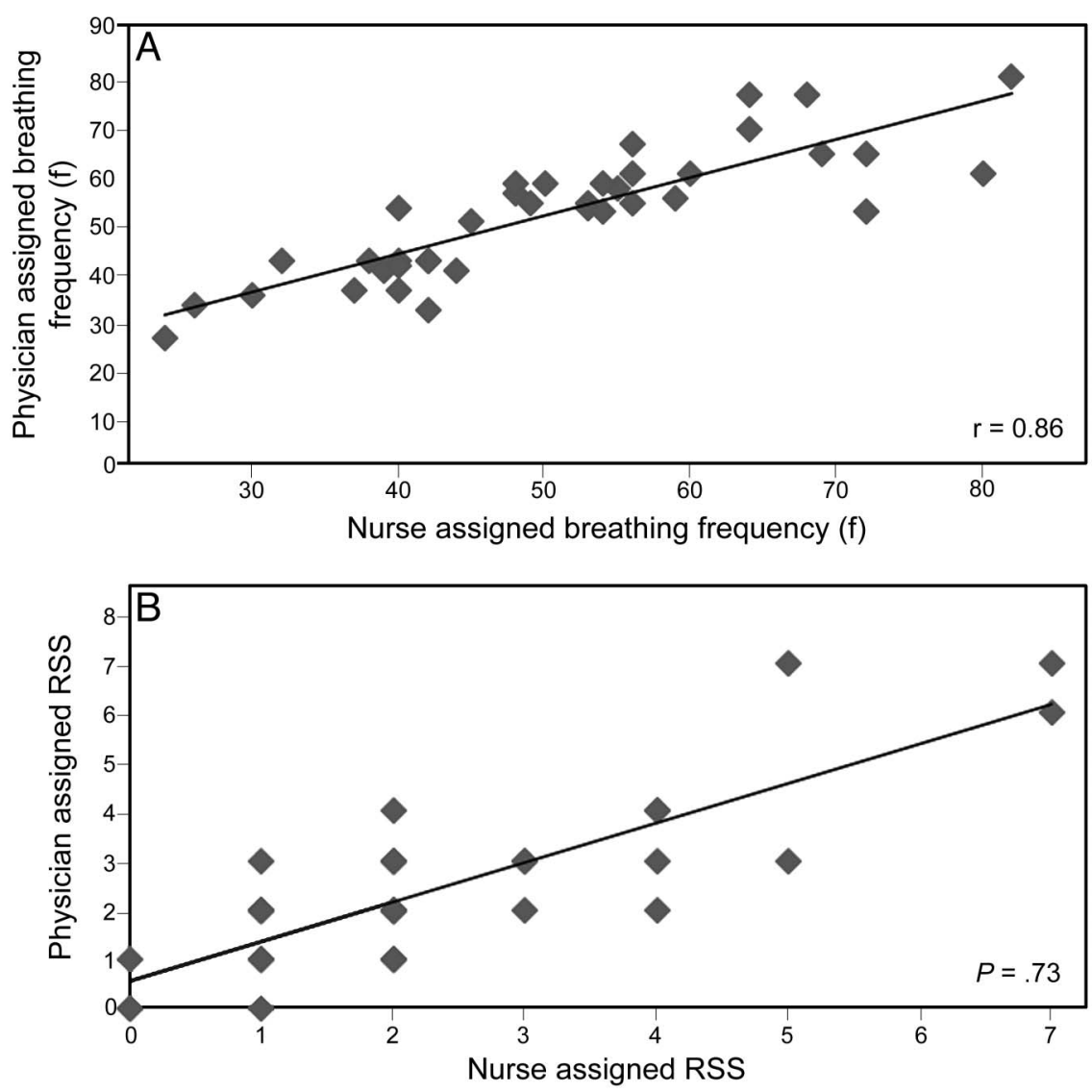

Fig. 3. Scatter plots comparing respiratory assessments between physicians and nurses. A: Breathing frequency of the 38 subjects in the NICU ranged from 24 to 80 breaths/min. The Pearson correlation coefficient for breathing frequency between physician and nurse was 0.86. B: Respiratory severity score (RSS) of these same subjects ranged from 0 to 7 as determined by the physician on duty. Nurses had the same score as the physician $45 \%$ of the time, were within 1 point $87 \%$ of the time, and were within 2 points $100 \%$ of the time. The Spearman correlation coefficient for respiratory severity scoring between physician and nurse was 0.73 .

\section{Discussion}

The majority of preterm neonates with respiratory distress are born in low-income and middle-income countries. ${ }^{17}$ These neonates are at high risk of death because most are born in settings with poor coverage of maternal antenatal steroid therapy for preterm labor and which lack surfactant treatment and mechanical ventilation for neonatal respiratory distress. ${ }^{18}$ The use of CPAP is effective for the treatment of respiratory failure in high-income countries, ${ }^{5-7}$ but there are few reports of the use of CPAP in low-income settings. ${ }^{10-12,19}$ Bubble CPAP is an attractive device for treating neonates with respiratory distress due to its simple design, low cost, and potential to improve survival rates in settings lacking mechanical ventilation. However, in an effort to provide bubble CPAP to neonates with respiratory distress in low-income and middle-income countries, health-care providers may use untested homemade devices despite uncertainty about the devices' ability to deliver CPAP reliably. ${ }^{19}$ A strength of our study is that the bubble CPAP device used at Kiwoko Hospital was shown to produce reliable CPAP pressures at the nasal cannula interface that corresponded to water column levels.

Our descriptive study demonstrates that after a short training period, a low-cost bubble CPAP device coupled with RSS assessments can be implemented and used by nurses and physicians to treat neonates with respiratory distress in low-income and middle-income countries. Based on the NICU physicians' judgment, only neonates thought to benefit from CPAP, with a high likelihood of dying without CPAP, were eligible for bubble CPAP treatment. Initiation of bubble CPAP was associated with decreased breathing frequency and an increase in $\mathrm{S}_{\mathrm{pO}_{2}}$ over the course of treatment in surviving infants.

Additionally, our study demonstrates that the RSS is easily taught to NICU physicians and nurses and that this assessment skill is retained over time. Three months after initial instruction, nurses and physicians had a similar inter-rater reliability for the RSS comparable to the breathing frequency, a more traditional objective assessment. 


\section{Bubble CPAP in a RuRal Ugandan NICU}

Table 1. Characteristics of Neonates Treated With Bubble CPAP

\begin{tabular}{|c|c|c|c|c|c|c|c|c|c|c|c|}
\hline \multicolumn{4}{|c|}{ Demographics } & \multicolumn{4}{|c|}{ Status Before CPAP } & \multicolumn{4}{|c|}{ CPAP Treatment } \\
\hline Subject & $\begin{array}{l}\text { CGA } \\
\text { (wk) }\end{array}$ & $\begin{array}{l}\text { Weight } \\
\text { (g) }\end{array}$ & Diagnosis & $\begin{array}{c}\mathrm{NC} \mathrm{O}_{2} \\
(\%, \mathrm{~L} / \mathrm{min})\end{array}$ & $\mathrm{S}_{\mathrm{pO}_{2}}(\%)$ & $\begin{array}{c}\mathrm{f} \\
\text { (breaths/min) }\end{array}$ & $\begin{array}{l}\text { RSS } \\
\text { Score }\end{array}$ & $\begin{array}{l}\text { Age } \\
\text { (d) }\end{array}$ & $\begin{array}{c}\text { Peak } \\
\left(\mathrm{cm} \mathrm{H}_{2} \mathrm{O}\right)\end{array}$ & $\begin{array}{l}\text { Duration } \\
\text { (d) }\end{array}$ & $\begin{array}{l}\text { Weaned/ } \\
\text { Stopped }\end{array}$ \\
\hline 1 & 31 & 1,240 & RDS & $100, \mathrm{NA}$ & 97 & 70 & 6 & 2 & 5 & 5 & $\mathrm{NC}$ \\
\hline 2 & 33 & 2,300 & RDS & 100, NA & 85 & 100 & 7 & 0 & 8 & 5 & Death \\
\hline 3 & 30 & 1,480 & RDS & $100,0.25$ & 93 & 68 & 7 & 2 & 5 & 2 & Death \\
\hline 4 & 28 & 1,070 & RDS & 100,2 & 90 & 50 & 9 & 0 & 6 & 0.5 & Death \\
\hline 5 & 30 & 1,300 & RDS & 100,2 & 90 & 80 & 9 & 0 & 5 & 2 & Death \\
\hline 6 & Term & 2,780 & Asphyxia & 100,2 & 94 & 60 & 9 & 0 & 5 & 0.1 & Air \\
\hline 7 & 30 & 1,360 & RDS & 100,2 & 92 & 80 & 9 & 2 & 5 & 2 & Death \\
\hline 8 & 30 & 1,240 & RDS & 100,5 & 100 & 70 & 7 & 2 & 5 & 3 & $\mathrm{NC}$ \\
\hline 9 & 32 & 1,680 & RDS & 100,2 & 95 & 70 & 7 & 0 & 5 & 2 & $\mathrm{NC}$ \\
\hline 10 & 32 & 1,800 & RDS & 100,2 & 88 & 80 & 8 & 0 & 5 & 1 & Air \\
\hline 11 & 40 & 3,460 & Asphyxia & 100,5 & 85 & 10 & Gasping & 3 & 6 & 0.5 & Death \\
\hline 12 & Term & NA & Asphyxia & 100,5 & 80 & 60 & 7 & 0 & 5 & 0.5 & $\mathrm{NC}$ \\
\hline 13 & 32 & 1,560 & RDS & 100,2 & 85 & 60 & 8 & 0 & 6.5 & 5 & $\mathrm{NC}$ \\
\hline 14 & 32 & 1,690 & RDS & 100,3 & 100 & 70 & NA & 0 & 5 & 5 & $\mathrm{NC}$ \\
\hline 15 & Term & 2,900 & Asphyxia & 100,5 & 92 & 80 & 7 & 0 & 7 & 2 & Death \\
\hline 16 & 32 & 1,360 & RDS & 100,5 & 85 & 50 & 4 & 3 & 6 & 3 & Death \\
\hline 17 & 29 & 1,240 & RDS & 100,1 & 92 & 60 & 6 & 0 & 6 & 5 & $\mathrm{NC}$ \\
\hline 18 & Term & 2,610 & Asphyxia & NA, 5 & NA & NA & 7 & 0 & 5 & 0.5 & Death \\
\hline 19 & 34 & 1,900 & RDS & $100,0.25$ & 100 & 70 & 8 & 0 & 5 & 8 & $\mathrm{NC}$ \\
\hline 20 & 32 & 1,520 & RDS & 100,2 & 95 & 80 & 8 & 0 & 5 & 4 & $\mathrm{NC}$ \\
\hline 21 & 30 & 1,300 & RDS & None & 93 & 48 & 8 & 0 & 4 & 2 & $\mathrm{NC}$ \\
\hline \multicolumn{12}{|c|}{$\begin{array}{l}\mathrm{CGA}=\text { corrected gestational age } \\
\mathrm{NC}=\text { nasal cannula } \\
\mathrm{f}=\text { breathing frequency } \\
\mathrm{RSS}=\text { respiratory severity score } \\
\mathrm{RDS}=\text { respiratory distress syndrome } \\
\mathrm{NA}=\text { not available }\end{array}$} \\
\hline
\end{tabular}

Table 2. Respiratory-Related Measurements at Different Time Points in Neonates Treated With Bubble CPAP

\begin{tabular}{lcccc}
\hline \hline & Baseline & $2-4 \mathrm{~h}$ & 12-24h & End Point \\
\hline Mean time, $\mathrm{h}$ & 0 & $3.5 \pm 1.8(20)$ & $18.3 \pm 5(19)$ & $79.1 \pm 42.8(15)$ \\
Breathing frequency, breaths/min & $65.8 \pm 18.3(20)$ & $64.3 \pm 17.2(19)$ & $64.7 \pm 15(17)$ & $56.2 \pm 16.4(12)$ \\
$\mathrm{S}_{\mathrm{pO}_{2}, \%}$ & $86 \pm 11(20)$ & $95 \pm 9(20)$ & $96 \pm 5(18)$ & $86 \pm 26(13)$ \\
$\mathrm{RSS}$ & $7.4 \pm 1.26(19)$ & $5.2 \pm 2.26(18)$ & $4.9 \pm 2.74(16)$ & $3.5 \pm 1.85(13)$ \\
$\mathrm{CPAP}, \mathrm{cm} \mathrm{H} \mathrm{O}$ & $4.9 \pm 0.36(21)$ & $5.2 \pm 0.49(20)$ & $5.3 \pm 0.84(18)$ & $5.3 \pm 1.32(13)$ \\
Flow, L/min & $5.1 \pm 1.2(21)$ & $5.2 \pm 1.09(20)$ & $5.4 \pm 1.72(18)$ & $5.5 \pm 1.76(13)$ \\
& & & & \\
Values are expressed as mean $\pm \mathrm{SE}(n)$. & & & & \\
RSS = respiratory severity score & & & & \\
\hline
\end{tabular}

The RSS has been used in other low-income/middleincome hospitals to guide CPAP treatment, ${ }^{17,20}$ and our study suggests this is a reliable tool for hospitals lacking blood gas machines or $\mathrm{x}$-ray capability. However, additional follow-up testing of health-care providers is needed to establish long-term retention of reliable RSS assessment skills, and correlation with blood gas measurements is warranted to establish accuracy in detecting respiratory failure.
Published reports of neonates treated with CPAP in low-income and middle-income countries are limited and heterogeneous, with no large randomized control trials available. ${ }^{10-12,21}$ In a study from South Africa, Pieper et al ${ }^{21}$ reported the use of nasal CPAP in 11 extremely lowbirthweight infants with respiratory distress following birth compared with 10 control infants. Infants treated with nasal CPAP had a mean birthweight of $915 \mathrm{~g}$ and a mean gestational age of 28.6 weeks. At discharge from the 


\section{Bubble CPAP in a RuRAl Ugandan NICU}

hospital, 45\% (5/11) of infants in the nasal CPAP group survived versus $20 \%(2 / 10)$ in the control group. In a study from Malawi, van den Heuvel et al ${ }^{11}$ described the implementation of bubble CPAP in a busy teaching hospital NICU (3,500 admissions/y) with very limited resources and demonstrated that bubble CPAP could be used independently by nurses after a short training period. In this study, 3 of 5 neonates treated with bubble CPAP who met the inclusion criteria (birthweight between 1 and $2.5 \mathrm{~kg}$ and persistent respiratory distress $4 \mathrm{~h}$ after birth) survived compared with none of the 6 neonates treated with bubble CPAP who did not meet the selection criteria. In a retrospective study based in Fiji, Koyamaibole et al ${ }^{10}$ described the feasibility of nurses implementing bubble CPAP and demonstrated a 50\% reduction in the need for mechanical ventilation in the 18 months after the introduction of bubble CPAP. This study did not report nurse-to-neonate ratios and was done in an NICU with mechanical ventilation. Additionally, this study was conducted in a setting in which $98 \%$ of mothers delivered in a health facility, and all health care, including transport, was free and funded by the Fiji Government. Kawaza et $\mathrm{al}^{12}$ recently reported the implementation of a novel low-cost bubble CPAP system (approximately $\$ 350$ United States dollars) for low-resource settings at a referral hospital in Malawi. In this nonrandomized study including a total of 87 neonates (62 CPAP subjects and 25 control subjects), the authors reported an overall $27 \%$ improvement in survival in the CPAP group, and this effect was greatest for very lowbirthweight infants and those with RDS and sepsis. This study demonstrated the efficacy of bubble CPAP in a lowresource setting and highlights the need for further studies in a variety of low-income/middle-income settings, including rural areas.

Our descriptive study has many limitations. We focused on the feasibility of implementing bubble CPAP in a rural low-income/middle-income setting; this was not a randomized trial aimed at testing the effectiveness of this noncommercial bubble CPAP device. Although a randomized trial of bubble CPAP with established entry criteria and a standardized management protocol may provide a more accurate measure of survival, this type of study may be challenging because nurses and physicians may not be willing to withhold CPAP in settings in which this modality could potentially save lives. ${ }^{11,21}$ Finally, we did not have blood gas measurements or x-rays to correlate with the RSS, which, if available, may have influenced who received CPAP and outcomes.

In future studies, it will be important to study multiple factors that may impact outcomes in sick neonates treated with bubble CPAP. Setting up and maintaining bubble CPAP require additional human resources and may affect equitable distribution of care. In the study by van den Heuvel et $\mathrm{al}^{11}$ from Malawi, only 2 nurses were available during the day and night to cover 73 neonatal beds in the Queen Elizabeth Central Hospital in Blantyre. Determining whether nurse and physician time allocation to a sick infant on bubble CPAP detracts from the care of other infants in the NICU, although potentially difficult to quantify, may be imperative to prevent an overall negative effect on neonatal outcomes and to establish targets for workforce staffing with scale up of this intervention. Establishing CPAP selection criteria along with parameters for weaning may be important in guiding NICU providers at to which neonates to treat with CPAP and for optimal respiratory management, particularly when resources are limited.

\section{Conclusions}

At Kiwoko Hospital, NICU physicians and nurses had similar RSS assessments of neonates with respiratory distress. The effectiveness of the RSS tool in assessing respiratory severity in neonates needs further validation, but it is feasible to implement in a low-income/middle-income setting. Future studies are needed to determine the effectiveness of bubble CPAP on overall neonatal survival in low-income/middle-income settings.

\section{REFERENCES}

1. Lozano R, Naghavi M, Foreman K, Lim S, Shibuya K, Aboyans V, et al. Global and regional mortality from 235 causes of death for 20 age groups in 1990 and 2010: a systematic analysis for the Global Burden of Disease Study 2010. Lancet 2012;380(9859):2095-2128.

2. Schmölzer GM, Kumar M, Pichler G, Aziz K, O'Reilly M, Cheung PY. Non-invasive versus invasive respiratory support in preterm infants at birth: systematic review and meta-analysis. BMJ 2013; 347:f5980.

3. Polin RA, Carlo WA. Surfactant replacement therapy for preterm and term neonates with respiratory distress. Pediatrics 2014;133(1): 156-163.

4. Henderson-Smart DJ, Wilkinson A, Raynes-Greenow CH. Mechanical ventilation for newborn infants with respiratory failure due to pulmonary disease. Cochrane Database Syst Rev 2002;(4):CD002770.

5. Polin RA, Sahni R. Newer experience with CPAP. Semin Neonatol 2002;7(5):379-389.

6. Sekar KC, Corff KE. To tube or not to tube babies with respiratory distress syndrome. J Perinatol 2009;29(Suppl 2):S68-S72.

7. Ho JJ, Subramaniam P, Henderson-Smart DJ, Davis PG. Continuous distending pressure for respiratory distress syndrome in preterm infants. Cochrane Database Syst Rev 2002(2):CD002271.

8. DiBlasi RM. Neonatal noninvasive ventilation techniques: do we really need to intubate? Respir Care 2011;56(9):1273-1294; discussion 1295-1277.

9. Duke T, Peel D, Graham S, Howie S, Enarson PM, Jacobson R. Oxygen concentrators: a practical guide for clinicians and technicians in developing countries. Ann Trop Paediatr 2010;30(2):87-101.

10. Koyamaibole L, Kado J, Qovu JD, Colquhoun S, Duke T. An evaluation of bubble-CPAP in a neonatal unit in a developing country: effective respiratory support that can be applied by nurses. J Trop Pediatr 2006;52(4):249-253. 


\section{Bubble CPAP in a RuRal Ugandan NICU}

11. van den Heuvel M, Blencowe $\mathrm{H}$, Mittermayer $\mathrm{K}$, Rylance $\mathrm{S}$, Couperus A, Heikens GT, Bandsma RH. Introduction of bubble CPAP in a teaching hospital in Malawi. Ann Trop Paediatr 2011; 31(1):59-65.

12. Kawaza K, Machen HE, Brown J, Mwanza Z, Iniguez S, Gest A, et al. Efficacy of a low-cost bubble CPAP system in treatment of respiratory distress in a neonatal ward in Malawi. PLoS ONE 2014; 9(1):e86327.

13. Diblasi RM. Nasal continuous positive airway pressure (CPAP) for the respiratory care of the newborn infant. Respir Care 2009;54(9): 1209-1235.

14. Silverman WA, Andersen DH. A controlled clinical trial of effects of water mist on obstructive respiratory signs, death rate and necropsy findings among premature infants. Pediatrics 1956;17(1): $1-10$.

15. Uganda Bureau of Statistics. 2002 Uganda population and housing census: population size and distribution 2002. http://www.ubos.org/ onlinefiles/uploads/ubos/pdf\%20documents/2002\%20CensusPopnSizeGrowthAnalyticalReport.pdf. Accessed October 9, 2014.
16. Uganda Bureau of Statistics. Uganda demographic and health survey 2011. http://www.ubos.org/onlinefiles/uploads/ubos/UDHS/ UDHS2011.pdf. Accessed October 9, 2014.

17. Jamison DT, Feachem RG, Makgoba MW, Bos ER, Baingana FK, Hofman KJ, and Rogo KO, editors. Disease and Mortality in SubSaharan Africa, 2nd edition. Washington, DC: World Bank; 2006.

18. Lawn JE, Kerber K, Enweronu-Laryea C, Cousens S. 3.6 million neonatal deaths-what is progressing and what is not? Semin Perinatol 2010;34(6):371-386.

19. Daga S, Mhatre S, Borhade A, Khan D. Home-made continuous positive airways pressure device may reduce mortality in neonates with respiratory distress in low-resource setting. J Trop Pediatr 2014; 60(5):343-347.

20. Tagare A, Kadam S, Vaidya U, Pandit A, Patole S. Bubble CPAP versus ventilator CPAP in preterm neonates with early onset respiratory distress - a randomized controlled trial. J Trop Pediatr 2013; 59(2):113-119.

21. Pieper CH, Smith J, Maree D, Pohl FC. Is nCPAP of value in extreme preterms with no access to neonatal intensive care? J Trop Pediatr 2003;49(3):148-152. 